Network Working Group

Request for Comments: 2001

W. Stevens

NOAO

Category: Standards Track

January 1997

TCP Slow Start, Congestion Avoidance,
Fast Retransmit, and Fast Recovery Algorithms

Status of this Memo

This document specifies an Internet standards track protocol for the Internet community, and requests discussion and suggestions for improvements. Please refer to the current edition of the "Internet Official Protocol Standards" (STD 1) for the standardization state and status of this protocol. Distribution of this memo is unlimited.

Abstract

Modern implementations of TCP contain four intertwined algorithms that have never been fully documented as Internet standards: slow start, congestion avoidance, fast retransmit, and fast recovery. and [3] provide some details on these algorithms, [4] provides examples of the algorithms in action, and [5] provides the source code for the 4.4BSD implementation. RFC 1122 requires that a TCP must implement slow start and congestion avoidance (Section 4.2.2.15 of [1]), citing [2] as the reference, but fast retransmit and fast recovery were implemented after RFC 1122. The purpose of this document is to document these four algorithms for the Internet.

Acknowledgments

Much of this memo is taken from "TCP/IP Illustrated, Volume 1: The Protocols" by W. Richard Stevens (Addison-Wesley, 1994) and "TCP/IP Illustrated, Volume 2: The Implementation" by Gary R. Wright and W. Richard Stevens (Addison-Wesley, 1995). This material is used with the permission of Addison-Wesley. The four algorithms that are described were developed by Van Jacobson.

1. Slow Start

Old TCPs would start a connection with the sender injecting multiple segments into the network, up to the window size advertised by the receiver. While this is OK when the two hosts are on the same LAN, if there are routers and slower links between the sender and the receiver, problems can arise. Some intermediate router must queue the packets, and it's possible for that router to run out of space. [2] shows how this naive approach can reduce the throughput of a TCP connection drastically. 
The algorithm to avoid this is called slow start. It operates by observing that the rate at which new packets should be injected into the network is the rate at which the acknowledgments are returned by the other end.

Slow start adds another window to the sender's TCP: the congestion window, called "cwnd". When a new connection is established with a host on another network, the congestion window is initialized to one segment (i.e., the segment size announced by the other end, or the default, typically 536 or 512). Each time an ACK is received, the congestion window is increased by one segment. The sender can transmit up to the minimum of the congestion window and the advertised window. The congestion window is flow control imposed by the sender, while the advertised window is flow control imposed by the receiver. The former is based on the sender's assessment of perceived network congestion; the latter is related to the amount of available buffer space at the receiver for this connection.

The sender starts by transmitting one segment and waiting for its ACK. When that $A C K$ is received, the congestion window is incremented from one to two, and two segments can be sent. When each of those two segments is acknowledged, the congestion window is increased to four. This provides an exponential growth, although it is not exactly exponential because the receiver may delay its ACKs, typically sending one ACK for every two segments that it receives.

At some point the capacity of the internet can be reached, and an intermediate router will start discarding packets. This tells the sender that its congestion window has gotten too large.

Early implementations performed slow start only if the other end was on a different network. Current implementations always perform slow start.

\section{Congestion Avoidance}

Congestion can occur when data arrives on a big pipe (a fast LAN) and gets sent out a smaller pipe (a slower WAN). Congestion can also occur when multiple input streams arrive at a router whose output capacity is less than the sum of the inputs. Congestion avoidance is a way to deal with lost packets. It is described in [2].

The assumption of the algorithm is that packet loss caused by damage is very small (much less than 1\%), therefore the loss of a packet signals congestion somewhere in the network between the source and destination. There are two indications of packet loss: a timeout occurring and the receipt of duplicate ACKs. 
Congestion avoidance and slow start are independent algorithms with different objectives. But when congestion occurs TCP must slow down its transmission rate of packets into the network, and then invoke slow start to get things going again. In practice they are implemented together.

Congestion avoidance and slow start require that two variables be maintained for each connection: a congestion window, cwnd, and a slow start threshold size, ssthresh. The combined algorithm operates as follows :

1. Initialization for a given connection sets cwnd to one segment and ssthresh to 65535 bytes.

2. The TCP output routine never sends more than the minimum of cwnd and the receiver's advertised window.

3. When congestion occurs (indicated by a timeout or the reception of duplicate ACKS), one-half of the current window size (the minimum of cwnd and the receiver's advertised window, but at least two segments) is saved in ssthresh. Additionally, if the congestion is indicated by a timeout, cwnd is set to one segment (i.e., slow start).

4. When new data is acknowledged by the other end, increase cwnd, but the way it increases depends on whether TCP is performing slow start or congestion avoidance.

If cwnd is less than or equal to ssthresh, TCP is in slow start; otherwise TCP is performing congestion avoidance. Slow start continues until TCP is halfway to where it was when congestion occurred (since it recorded half of the window size that caused the problem in step 2), and then congestion avoidance takes over.

Slow start has cwnd begin at one segment, and be incremented by one segment every time an ACK is received. As mentioned earlier, this opens the window exponentially: send one segment, then two, then four, and so on. Congestion avoidance dictates that cwnd be incremented by segsize*segsize/cwnd each time an ACK is received, where segsize is the segment size and cwnd is maintained in bytes. This is a linear growth of cwnd, compared to slow start's exponential growth. The increase in cwnd should be at most one segment each round-trip time (regardless how many ACKs are received in that RTT), whereas slow start increments cwnd by the number of ACKs received in a round-trip time. 
Many implementations incorrectly add a small fraction of the segment size (typically the segment size divided by 8) during congestion avoidance. This is wrong and should not be emulated in future releases.

3. Fast Retransmit

Modifications to the congestion avoidance algorithm were proposed in 1990 [3]. Before describing the change, realize that TCP may generate an immediate acknowledgment (a duplicate ACK) when an outof-order segment is received (Section 4.2.2.21 of [1], with a note that one reason for doing so was for the experimental fastretransmit algorithm). This duplicate ACK should not be delayed. The purpose of this duplicate ACK is to let the other end know that a segment was received out of order, and to tell it what sequence number is expected.

Since TCP does not know whether a duplicate ACK is caused by a lost segment or just a reordering of segments, it waits for a small number of duplicate ACKs to be received. It is assumed that if there is just a reordering of the segments, there will be only one or two duplicate ACKs before the reordered segment is processed, which will then generate a new ACK. If three or more duplicate ACKs are received in a row, it is a strong indication that a segment has been lost. TCP then performs a retransmission of what appears to be the missing segment, without waiting for a retransmission timer to expire.

\section{Fast Recovery}

After fast retransmit sends what appears to be the missing segment, congestion avoidance, but not slow start is performed. This is the fast recovery algorithm. It is an improvement that allows high throughput under moderate congestion, especially for large windows.

The reason for not performing slow start in this case is that the receipt of the duplicate ACKs tells TCP more than just a packet has been lost. Since the receiver can only generate the duplicate ACK when another segment is received, that segment has left the network and is in the receiver's buffer. That is, there is still data flowing between the two ends, and TCP does not want to reduce the flow abruptly by going into slow start.

The fast retransmit and fast recovery algorithms are usually implemented together as follows. 
1. When the third duplicate $A C K$ in a row is received, set ssthresh to one-half the current congestion window, cwnd, but no less than two segments. Retransmit the missing segment. Set cwnd to ssthresh plus 3 times the segment size. This inflates the congestion window by the number of segments that have left the network and which the other end has cached (3).

2. Each time another duplicate ACK arrives, increment cwnd by the segment size. This inflates the congestion window for the additional segment that has left the network. Transmit a packet, if allowed by the new value of cwnd.

3. When the next ACK arrives that acknowledges new data, set cwnd to ssthresh (the value set in step 1). This ACK should be the acknowledgment of the retransmission from step 1 , one round-trip time after the retransmission. Additionally, this ACK should acknowledge all the intermediate segments sent between the lost packet and the receipt of the first duplicate ACK. This step is congestion avoidance, since TCP is down to one-half the rate it was at when the packet was lost.

The fast retransmit algorithm first appeared in the 4.3BSD Tahoe release, and it was followed by slow start. The fast recovery algorithm appeared in the 4.3BSD Reno release.

\section{Security Considerations}

Security considerations are not discussed in this memo.

\section{References}

[1] B. Braden, ed., "Requirements for Internet Hosts -Communication Layers," RFC 1122, Oct. 1989.

[2] V. Jacobson, "Congestion Avoidance and Control," Computer Communication Review, vol. 18, no. 4, pp. 314-329, Aug. 1988. ftp://ftp.ee.lbl.gov/papers/congavoid.ps.z.

[3] V. Jacobson, "Modified TCP Congestion Avoidance Algorithm," end2end-interest mailing list, April 30, 1990. ftp://ftp.isi.edu/end2end/end2end-interest-1990.mail.

[4] W. R. Stevens, "TCP/IP Illustrated, Volume 1: The Protocols", Addison-Wesley, 1994.

[5] G. R. Wright, W. R. Stevens, "TCP/IP Illustrated, Volume 2: The Implementation", Addison-Wesley, 1995. 
Author's Address:

W. Richard Stevens

1202 E. Paseo del Zorro

Tucson, AZ 85718

Phone: 520-297-9416

EMail: rstevens@noao.edu

Home Page: http://www. noao.edu/〜rstevens 